OPEN

SUBJECT AREAS:

ENVIRONMENTAL

MONITORING

NANOPARTICLES

Received

18 February 2014

Accepted

23 April 2014

Published

15 May 2014

Correspondence and requests for materials should be addressed to

H.B.Z. (zeng.haibo.

nano@gmail.com)

\title{
Engineering surface states of carbon dots to achieve controllable luminescence for solid-luminescent composites and sensitive $\mathrm{Be}^{2+}$ detection
}

\author{
Xiaoming Li',2, Shengli Zhang', Sergei A. Kulinich ${ }^{3,4}$, Yanli Liu ${ }^{1,2}$ \& Haibo Zeng ${ }^{1,2}$
}

\begin{abstract}
'Institute of Optoelectronics \& Nanomaterials, College of Materials Science and Engineering, Nanjing University of Science and Technology, Nanjing 210094, China, ${ }^{2}$ College of Materials Science and Technology, Nanjing University of Aeronautics and Astronautics, Nanjing 210016, China, ${ }^{3}$ Institute of Innovative Science and Technology, Tokai University, Hiratsuka, Kanagawa 259-1292, Japan, ${ }^{4}$ School of Engineering and Applied Science, Aston University, Birmingham, B4 7ET, United Kingdom.
\end{abstract}

Luminescent carbon dots (L-CDs) with high quantum yield value (44.7\%) and controllable emission wavelengths were prepared via a facile hydrothermal method. Importantly, the surface states of the materials could be engineered so that their photoluminescence was either excitation-dependent or distinctly independent. This was achieved by changing the density of amino-groups on the L-CD surface. The above materials were successfully used to prepare multicolor L-CDs/polymer composites, which exhibited blue, green, and even white luminescence. In addition, the excellent excitation-independent luminescence of L-CDs prepared at low temperature was tested for detecting various metal ions. As an example, the detection limit of toxic $\mathrm{Be}^{2+}$ ions, tested for the first time, was as low as $23 \mu \mathrm{M}$.

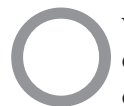

wing to their interesting and unique features, luminescent carbon dots (L-CDs) have recently drawn a lot of attention. Their merits, such as chemical stability, dispersibility in water, low photobleaching, biocompatibility and low toxicity, make them new-generation luminescent materials superior to conventionally used both fluorescent organic dyes and luminescent inorganic quantum dots (QDs) ${ }^{1,2}$. The potential applications of such materials cover such fields as bioimaging ${ }^{3,4}$, photocatalysis ${ }^{5}$, LED $^{6,7}$, energy conversion ${ }^{8}$ and sensing ${ }^{9-11}$, to mention just several. However, the lack of understanding of the photoluminescence (PL) origins in L-CDs hinders significantly the development and use of L-CD based materials with desired PL characteristics. Though many mechanisms have been proposed lately, e.g. those based on particle-size distribution, surface traps and quantum effect ${ }^{3,12}$, intramolecular $\mathrm{H}$-bonds ${ }^{10}$, charge transfer ${ }^{13}$, formation of aromatic molecules ${ }^{14}$ and radiation recombination of excitons ${ }^{15}$, there is no any universal interpretation in the literature thus far. For example, the PL peak position of L-CDs has been often reported to move with the change of excitation wavelength ${ }^{4,16}$, the phenomenon being closely related to the emission origins and not well understood yet. This excitation-dependent emission was frequently observed, whereas excitation-independent luminescence was seldom achieved in L-CDs, the reason for which still remaining unclear. While the understanding of emission origins and control over their luminescence are of extreme importance for more successful applications of L-CDs, up to now, most of the research has been focused on the improvement of their quantum yield $(\mathrm{QY})^{17-20}$.

In spite of the controversial character of the PL origins in L-CDs, the involvement of surface traps in the radiative transition of carbon dots has been widely accepted ${ }^{21-27}$. Surface groups, such as, e.g., C-O, C $=\mathrm{O}$ and $\mathrm{O}=\mathrm{C}-\mathrm{OH}$, can introduce trapping states with different energy levels, making L-CDs emit light that varies with excitation energy $\mathrm{y}^{28,29}$. Very recently, Yu and coauthors investigated the temperature dependence of L-CD luminescence and attributed the excitation-dependent emission to surface trap $s^{30}$. Based on this finding, we speculate that the excitation dependence of the L-CD luminescence could be controlled through engineering the above mentioned surface states of L-CDs, as shown in Figure 1. As an extreme case, if all surface states are completely passivated, the emission is believed to take place only through the radiative transition of $\mathrm{sp}^{2}$ carbon, probably the $\pi$ to $\pi^{*}$ transition, which will obviously result in excitation independence due to the single transition mode with a certain energy. Alternatively, if the surface states are not passivated, the emission modes related to $\mathrm{C}-\mathrm{O}, \mathrm{C}=\mathrm{O}$ and $\mathrm{O}=\mathrm{C}-\mathrm{OH}$ surface states with a series of specific energies will dominate the luminescence spectra depending 

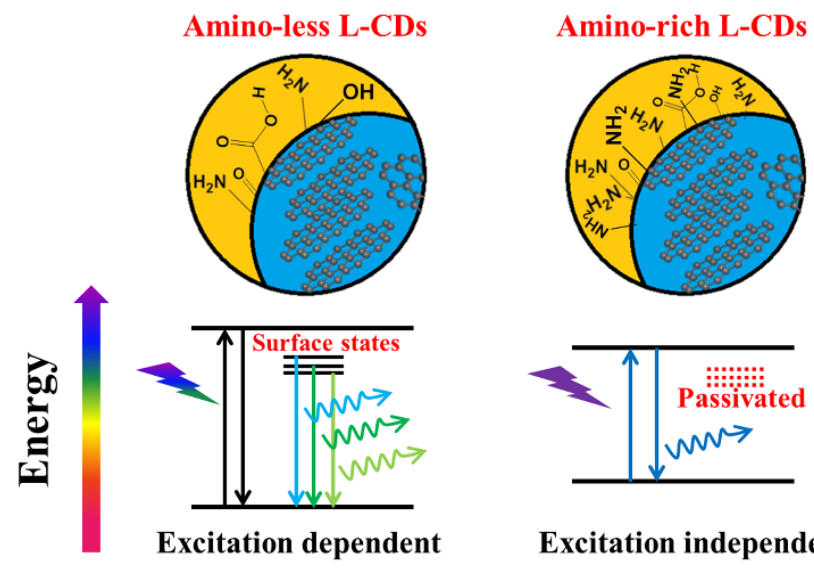

Excitation independent

Figure 1 Surface-state engineering of carbon dots with excitationdependent and excitation-independent luminescent properties. Carbon dots with various surface states (terminated with various groups) emit excitation-dependent light. L-CDs with surfaces rich in amino-groups exhibit excitation-independent behavior because of passivated surface states.

on excitation energy. In this case, L-CDs will demonstrate excitationdependent emission. Nitrogen containing organic compounds, especially those with short chains, are often used as effective passivating agents ${ }^{9,23,31}$. This implies that amino-groups could be effective candidate to achieve the controlled surface passivation of L-CDs and consequently - to determine the character of luminescence (making it excitation-dependent or independent).

In this work, we varied the passivation degree of surface traps on LCDs by changing their surface coverage with amino-groups, which allowed us to design and prepare materials with either excitationdependent or independent blue-green luminescence. A simple and environmentally friendly hydrothermal method using citric acid and urea as precursors was applied. During the reaction, urea not only acted as a carbon source, but also provided surface amino-groups, thus passivating surface traps of the product. The passivation degree was controlled by varying the process temperature as amino-groups are unstable and tend to leave the surface at higher temperatures. The L-CDs prepared at different temperatures automatically fell into two classes according to the character of their luminescence. The materials prepared at higher temperatures had more surface traps, and therefore their luminescence was excitation-dependent. In contrast, if prepared at lower temperatures, the dots were fully surface-passivated and emitted independently on excitation wavelength. As a next step, composites containing different L-CDs and polymers were fabricated and exhibited blue, green, and even white luminescence. Finally, the excellent excitation-independent luminescence of LCDs prepared at low temperature was tested for detecting various metal ions. As an example, the detection limit of toxic $\mathrm{Be}^{2+}$ ions, tested for the first time, was as low as $23 \mu \mathrm{M}$.

\section{Results}

Fabrication and structure. To obtain L-CDs with controlled aminopassivated surfaces, we followed the procedures schematically shown in Figure 2a. First, a polymerization reaction between citric acid and urea led to a polymer-like material, after which carbonization took place, forming L-CDs. This was confirmed by control samples containing only citric acid or urea which preserved their color unchanged up to temperatures above $240^{\circ} \mathrm{C}$. The surface states and their passivation with amino-groups depended on reaction temperature (the samples prepared at $130,160,200$ and $240^{\circ} \mathrm{C}$ were named as LCD-130, LCD-160, LCD-200 and LCD-240, respectively). Interestingly, at different temperatures, we obtained transparent blue and brown dispersions, as shown in Figure S1.
Samples with reaction temperatures above $200^{\circ} \mathrm{C}$ were brown, whereas those prepared below $200^{\circ} \mathrm{C}$ were blue in color, the latter color having been never reported before ${ }^{11,20,22}$. Being auxochromes, amino-groups should enhance the optical absorption of L-CDs, resulting in a deeper dispersion's color. Consequently, the L-CDs prepared at lower temperatures exhibited blue color, which confirms a higher density of amino-groups on their surface. The prepared liquid dispersions can be used as a blue fluorescent ink for painting, printing or writing, as shown in Figure S2. In addition, only dark-brown and sticky polymer-like materials could be obtained upon their drying in vacuum at $70^{\circ} \mathrm{C}$ for several days (Figure $2 \mathrm{a}$ ). Figure $2 \mathrm{~b}$ presents a transmission electron microscopy (TEM) image of L-CDs prepared at $160^{\circ} \mathrm{C}$. The dots are seen to be well dispersed and have a uniform size distribution with an average diameter of about $5 \mathrm{~nm}$ (Figure S3). The high-resolution TEM (HRTEM) image in Figure $2 \mathrm{~b}$ (inset), reveals the presence of both crystalline graphite and amorphous phases. Note that all the HRTEM, XRD and Raman results were well consistent, supporting the coexistence of both graphite and amorphous carbon phases in the final products, as shown in Figure $S 4^{15,32,33}$.

The FTIR spectra in Figures $2 \mathrm{c}$ and S5 display several peaks related to the surface of L-CDs. The absorption bands at $3000-3500 \mathrm{~cm}^{-1}$ are assigned to stretching vibrations of $-\mathrm{OH}$ and $\mathrm{H}_{2} \mathrm{~N}$ - groups, both of which make the dots hydrophilic and improve their stability and dispersibility in water. As seen in Figure $2 c$, the absorption intensity of the $\mathrm{N}-\mathrm{H}$ peak at $1549 \mathrm{~cm}^{-1}$ is stronger, while the absorption band of $\mathrm{C}=\mathrm{O}$ at $1648 \mathrm{~cm}^{-1}$ is hardly observed for LCD-160 when compared to LCD-240. This indicates that low-temperature samples possess more amino-groups. The C 1s XPS spectrum of a lowtemperature sample (Figure 2d) reveals different types of carbon atoms (see also Table S6): graphitic or aliphatic $(283.87 \mathrm{eV})$, oxygenated $(286.13 \mathrm{eV})$ and nitrous $(287.75 \mathrm{eV})^{22}$. The $\mathrm{N} 1 \mathrm{~s}$ spectrum of the same sample (Figure 2e) shows two peaks at $399.25 \mathrm{eV}$ and $400.49 \mathrm{eV}$, which are attributed to aromatic $\mathrm{N}$ and surface $\mathrm{H}_{2} \mathrm{~N}$ groups, respectively ${ }^{24}$. The aromatic $\mathrm{N}$ and $\mathrm{C}=\mathrm{O}$ bonds observed by XPS and FTIR indicate the formation of amide bonds, which is a result of dehydration and condensation reactions between carboxyl and amino-groups. Therefore, both XPS and FTIR results confirm that the surface coverage of samples with amino-groups differed depending on preparation temperature. They also indicate that urine molecules acted both as $\mathrm{N}$-dopant and surface passivating agent, thus considerably enhancing the fluorescence of prepared L-CDs ${ }^{13,23}$.

Optical properties. To explore further the effect of surface passivation on the optical properties of L-CDs, their PL and UVVis absorption spectra were studied. The UV-Vis absorption spectra are seen in Figures $3 \mathrm{a}$ and $\mathrm{c}$ to demonstrate a typical absorption band located at $234 \mathrm{~nm}$ and attributed to the $\mathrm{n}-\pi^{*}$ transition $^{34}$. In addition, a new absorption band was observed at about $335 \mathrm{~nm}$, which is assigned to the contribution of surface groups and the $\pi-\pi^{*}$ transition ${ }^{9}$. Notably, the $\pi-\pi^{*}$ transition of a low-temperature sample in Figure $3 \mathrm{a}$ is presented by a very strong and symmetric absorption peak, if compared to its $n-\pi^{*}$ transition, which was seldom observed for L-CDs. At the same time, similarly to most reports ${ }^{16,37}$, its high-temperature counterpart showed only an absorption shoulder with a low intensity (see Figure $3 \mathrm{c}$ ). Moreover, the $\pi-\pi^{*}$ peak in Figure $3 \mathrm{a}$ exhibits a redshift by $15 \mathrm{~nm}$ compared to that in Figure $3 c$, while the $n-\pi^{*}$ transition peak is steady for both samples. Thus, based on the above mentioned FTIR and XPS results (as well as theoretical calculations, see Figure S7 and Table S8), we conclude that it is the surface amino-groups that passivate the traps on the surface of LCD-160 and lead to a single transition mode. The latter transition mode manifests itself as a strong and symmetric $\pi-\pi^{*}$ absorption peak in Figure 3a. At the same time, because of a much smaller number of surface amino-groups, the dots in LCD-240 are not completely passivated. That is, they have various surface traps with different energy levels below HOMO, which introduce different 

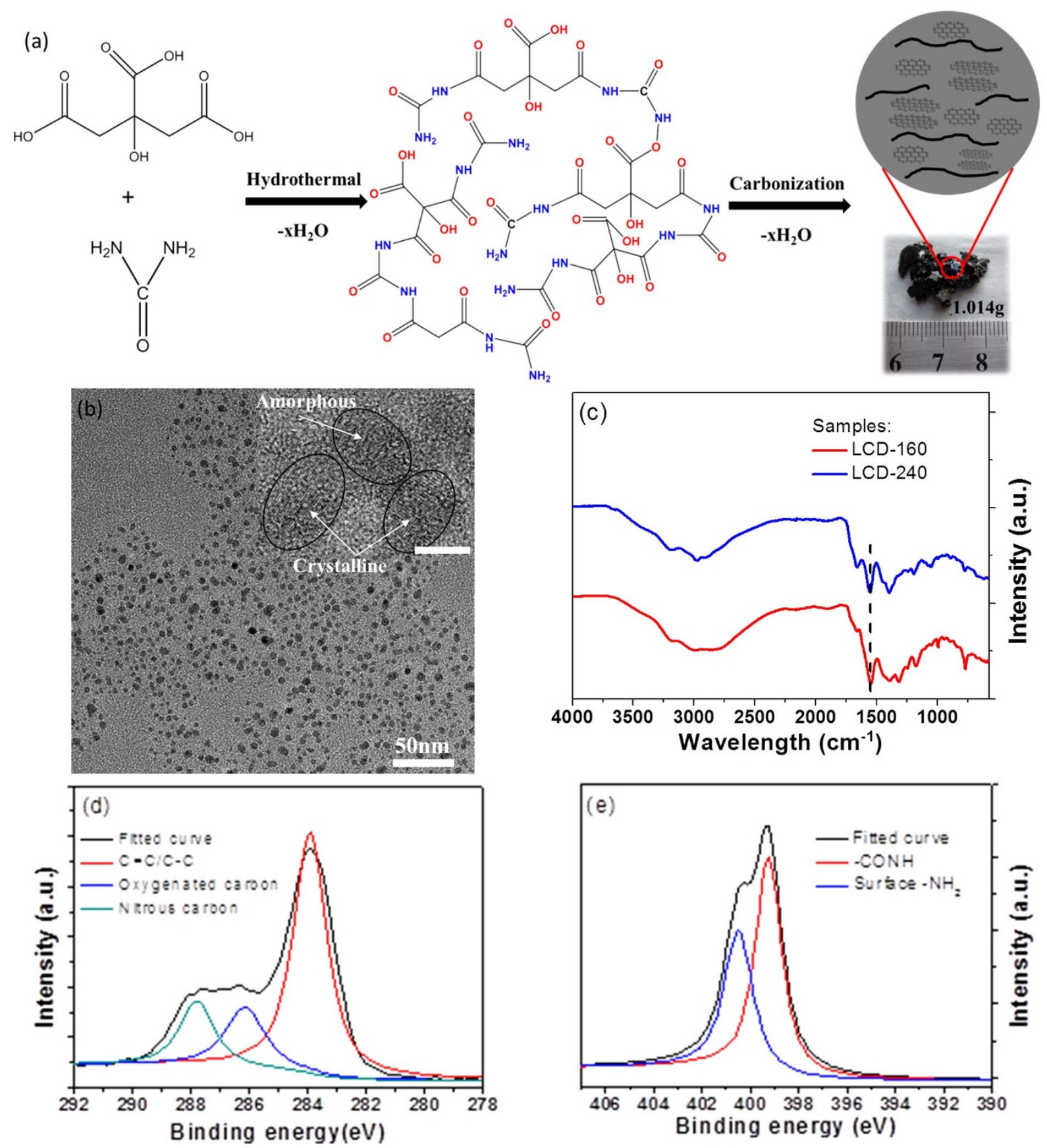

Figure $2 \mid$ (a) Schematic approach used to prepare carbon dots. The process includes precursor polymerization and subsequent carbonization. The image in the top right-hand side corner presents a schematic structure of the prepared dots, containing both polymer-like components (black stripes) and crystalline graphite segments (honeycomb clusters); a photograph of the product is also presented; (b) TEM image of L-CDs prepared at $160^{\circ} \mathrm{C}$, where the inset is an HRTEM image showing the existence of both amorphous and crystalline parts (scale bar indicates $10 \mathrm{~nm}$ ); (c) FTIR spectra of L-CDs fabricated at 160 and $240^{\circ} \mathrm{C}$; (d) C 1s and (e) $\mathrm{N} 1$ s XPS spectra of a sample prepared at $160^{\circ} \mathrm{C}$.

transition modes. The latter multi-absorption modes lead to a weak and asymmetric absorption shoulder in Figure 3c.

Since amino-groups are electron-donating, this can enhance the conjugation degree of $\mathrm{H}_{2} \mathrm{~N}$-passivated L-CDs. As a result, the probability of electron transition from the ground state to the lowest excited singlet state (and thus - optical absorption) should be higher $^{35}$. Therefore, the stronger absorption intensity of the $\pi-\pi^{*}$ transition in LCD-160 compared to that in LCD-240 is related to its larger number of surface amino-groups. In addition, the enhancement of electron cloud activity results in the narrowing of energy gap, which probably contributed to the redshift of absorption peak in Figure 3a. The redshift in Figure 3a was also supported by theoretical calculations, as shown in Figure S7 and Table S8. Compared with models that only contain carboxyl or hydroxyl-groups, the energy gaps of the systems decrease upon adding surface amino-groups. The energy levels indicated that amino-groups had a great contribution to the electron structure of L-CDs. All this indirectly confirms that lowtemperature samples possessed more surface amino-groups.

Figures 3b, d present the PL spectra of LCD-160 and LCD-240, respectively. In agreement with the above discussion, the emission peaks of LCD-160 are seen to be excitation-independent, while those of LCD-240 clearly shift toward longer wavelengths with the increase of excitation wavelength, which is explained by a single transition mode (LCD-160) or multiple transition modes (LCD-240) of the 

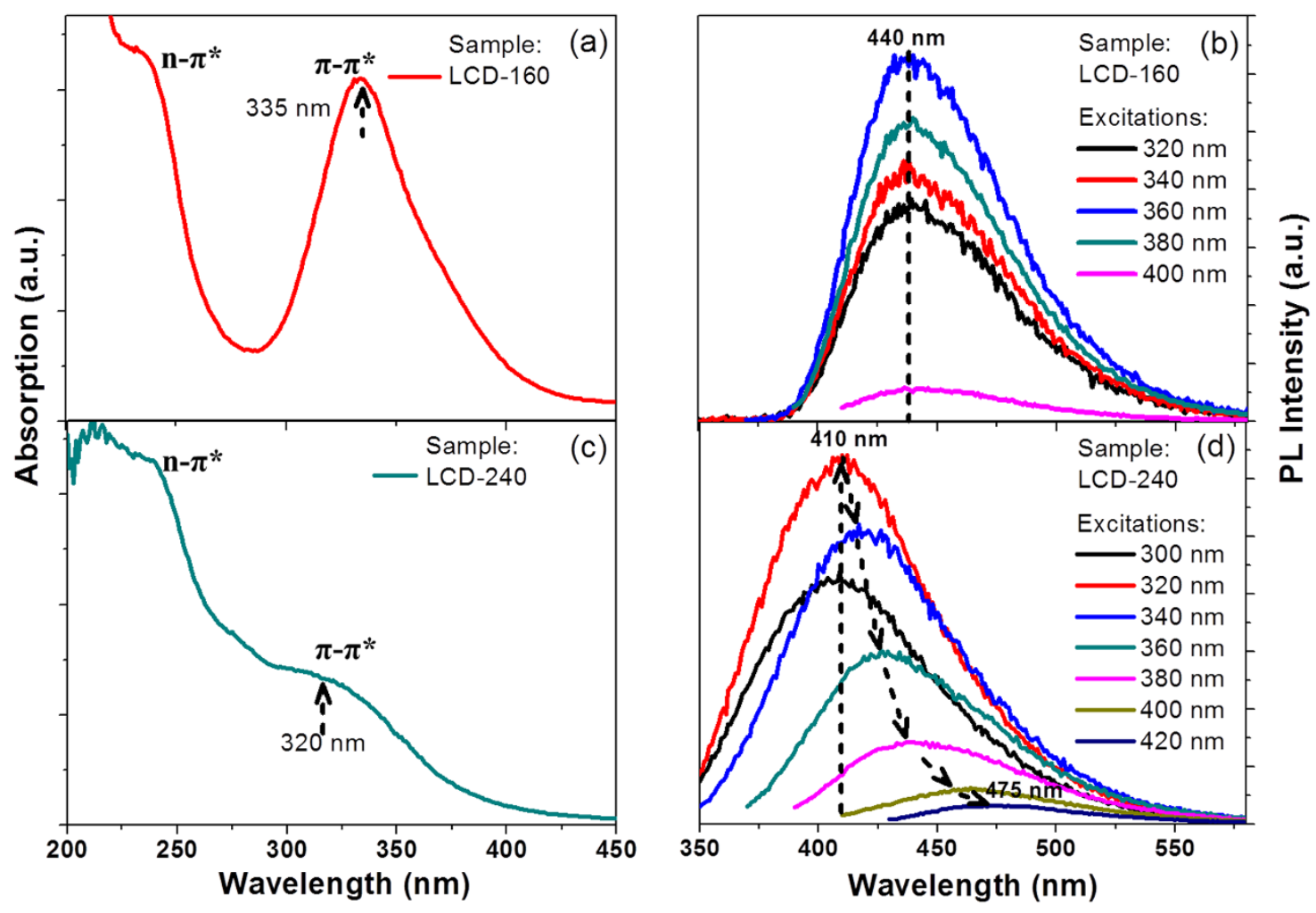

Figure $3 \mid \mathrm{UV}$-Vis absorption spectra of liquid samples prepared at 160 (a) and $240^{\circ} \mathrm{C}$ (c); PL emission peak positions are either independent (b) or dependent (d) of excitation wavelength for the materials prepared at 160 and $240^{\circ} \mathrm{C}$, respectively.

samples. The emission intensity of LCD-160 first increases, reaches a maximum at the excitation wavelength of $360 \mathrm{~nm}$, and then gradually decreases with a further increase in excitation wavelengths, while the peak position remains unchanged. As for LCD-240, the evolution of its emission with excitation wavelength can be separated into two regions. Below $320 \mathrm{~nm}$, the emission peaks keep their position mainly unchanged, after which they redshift with the increase of excitation wavelength. Surface states are known to introduce emission levels between $\mathrm{HOMO}$ and $\mathrm{LUMO}^{28,29}$. At different excitation wavelengths, different transition modes are known to have different probabilities. Each transition plays a dominating role at its corresponding excitation wavelength, contributing to the excitation dependence observed in the second region. Somewhat similar results were previously reported for $\mathrm{ZnO}$, where defect energy levels from interstitial zinc were found to contribute dramatically to the excitation dependence of product's $\mathrm{PL}^{36}$. The redshift of optimal excitation in Figure $3 \mathrm{~d}$ is in accordance with the absorption spectra in Figure 3c. To cross-check the above findings, two additional samples were prepared at 130 and $200^{\circ} \mathrm{C}$. As can be seen in Figure S9, the samples exhibited basically same results as those in Figures 3a-d: the absorption and PL spectra of the low-temperature (LCD-130) and hightemperature (LCD-200) samples were qualitatively close to those of LCD-160 and LCD-240, respectively. To the best of our knowledge, only several groups have previously reported on L-CDs with excitation-independent emission ${ }^{8,9,37-41}$, and control over excitation dependence or independence of L-CDs has been a great challenge thus far. In this work, we prepared L-CDs with different properties via engineering their surface states. By changing the density of surface amino-groups, we managed to modulate the surface states of the materials and prepare L-CDs either with excitation-dependent or independent optical properties.

Figure $4 \mathrm{a}$ presents time-resolved PL curves for LCD-160 and LCD-240 measured at room temperature. The decay curve for LCD-160 could be best fitted with a single-exponential function, while that for LCD-240 could be best fitted with a double-exponential function. Obviously, only one emission state dominated the PL properties of LCD-160 but two in LCD-240. The single emission state in LCD-160 brings about excitation-independent behavior ${ }^{42,43}$. For LCD-240, two dominating states are believed to correspond to the energy gap and surface state transitions. When excitation energy is higher than the energy gap, emission from the energy gap transition plays a leading role. When excitation energy is lower than the energy gap, surface state transitions participate in and contribute to the excitation-dependent behavior. Samples LCD-130 and LCD-200 (Figure S12) exhibited PL decay spectra similar to those of samples LCD-160 and LCD-240.

In addition, fluorescent molecules ${ }^{44}$ might form during the reaction processes. Whether the fluorescent molecules will influence the optical properties of our carbon dots is not very clear now. We are sure that we have eliminated their effect as much as possible. Luminescent carbon based materials with crystalline and welldefined structure will be presented in another work.

PL lifetime and Quantum yield. Detailed PL lifetimes and QY values of samples prepared at $130,160,200$ and $240^{\circ} \mathrm{C}$ are shown in Table 1. Figure $4 \mathrm{~b}$ shows the results of QY evaluation for LCD-160 and LCD-240 (data for quinine sulfate are also presented). The highest QY values (44.7\%) are seen to be demonstrated by sample LCD-160 whose PL was observed even under daylight excitation (Figure S12). Since electron-donating groups can enhance the conjugation degree of conjugated systems, this is expected to increase the transition probability from the ground state to the lowest excited singlet state and, thus, contribute to higher QY indirectly. Therefore, the QY of LCD-160 is higher than that of LCD-240, as the surface of the former sample is enriched with $\mathrm{H}_{2} \mathrm{~N}$-groups.

Thermal analyses. To confirm the pyrolysis of amino-groups at high temperatures, thermogravimetry (TG) measurements were carried 


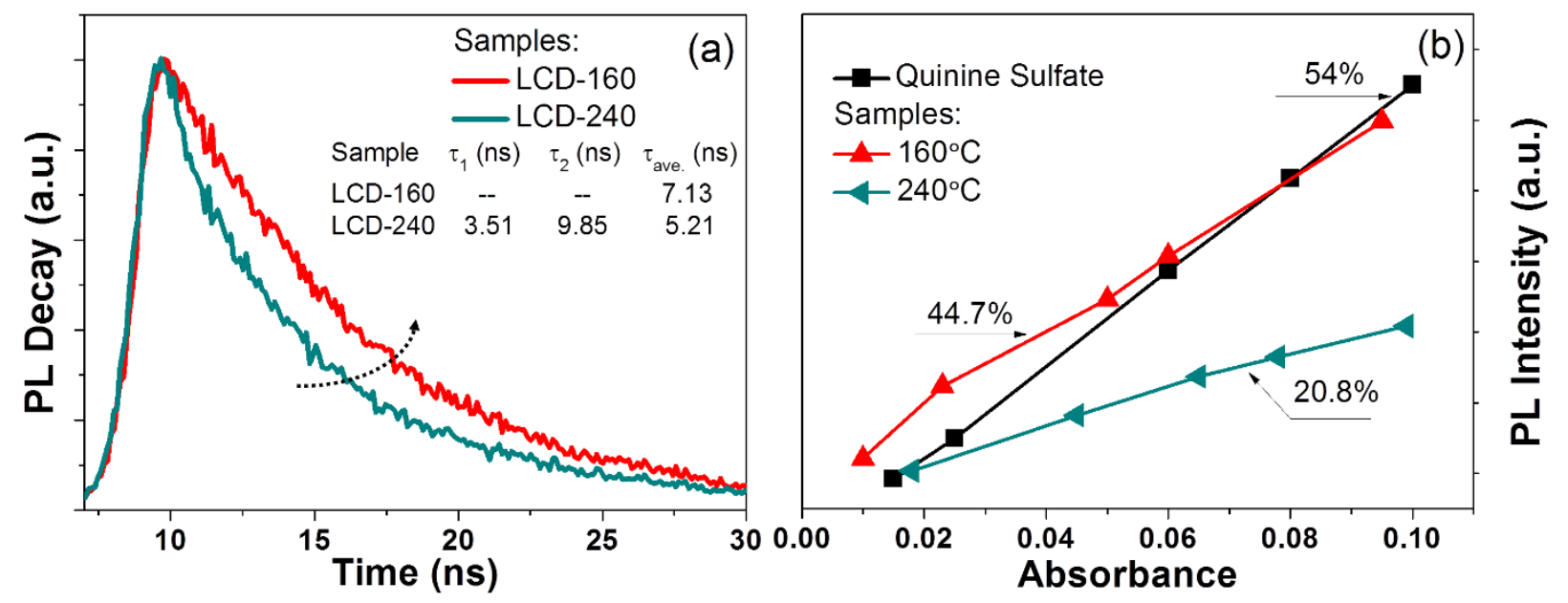

Figure $4 \mid$ (a) PL lifetime decay curves of the same samples; (b) Quantum yield of quinine sulfate and samples fabricated at 160 and $240{ }^{\circ} \mathrm{C}$.

out in $\mathrm{N}_{2}$. Figure 5 presents the obtained TG curves for four samples heated up to $600^{\circ} \mathrm{C}$. In agreement with previous reports, carbon cores are seen to survive within the whole range of applied temperatures ${ }^{45}$. Below $\sim 180^{\circ} \mathrm{C}$, all the samples demonstrated slopes with very similar behavior. At $180^{\circ} \mathrm{C}$, the curves of the low-temperature samples changed much more drastically if compared to their hightemperature counterparts, which is likely to indicate that the former samples began to lose their surface amino-groups around this temperature. The latter samples had much less amino-groups on their surface, and that is why their mass altered to a lesser extent at this temperature. A sample prepared at $180^{\circ} \mathrm{C}$ (results not shown here) also demonstrated trends similar to those of samples LCD-130 and LCD-160. Last but not the least, dilute dispersions of lowtemperature samples proved to be unstable over time. After three months of storage in air, their color changed from blue to brown though they were still luminescent (Figure S13). At the same time, the high-temperature samples remained mainly unchanged.

Multicolor composites. We also investigated the influence of ionic strength, $\mathrm{pH}$ and polarity of liquid media on the PL of prepared LCDs. Although all samples demonstrated a slight reduction in PL intensity, no trend was observed in the PL intensity variations or peak shape as the ionic strength of aqueous $\mathrm{KCl}$ medium was increased (Figure S14). This looks very promising as materials based on L-CDs are expected to be used in various environments and possibly with different electrolyte concentrations. Another important issue is the behavior of $\mathrm{PL}$ at different $\mathrm{pH}$ values (Figure S15). The as-prepared LCD-240 dispersion had a $\mathrm{pH}$ value of 8.21 . Its $\mathrm{PL}$ intensity increased with the increase of $\mathrm{pH}$ value from 2 to 4 and then remained stable at $\mathrm{pH}$ values larger than 5 . This observation is not consistent with the results of others who reported a monotonous increase of $\mathrm{PL}$ intensity with $\mathrm{pH}$ or an increase followed by a decrease $e^{25,40}$. The inconsistency might be explained by the instability of amino-groups at low $\mathrm{pH}$.

Upon drying, the samples could be redispersed successfully in water or another liquid without any aggregation, which is important

Table 1 | Photoluminescence lifetime and quantum yield (QY) of carbon colloids fabricated at $130,160,200$ and $240^{\circ} \mathrm{C}$. The highest QY was achieved at $160^{\circ} \mathrm{C}$

\begin{tabular}{lcccc} 
Sample & $\tau_{1}$ (ns) & $\tau_{2}$ (ns) & $\tau_{\text {ave }}$ (ns) & QY \% \\
\hline $130^{\circ} \mathrm{C}$ & 6.78 & -- & 6.78 & 35.4 \\
$160^{\circ} \mathrm{C}$ & 7.13 & -- & 7.13 & 44.7 \\
$200^{\circ} \mathrm{C}$ & 2.81 & 10.41 & 6.12 & 28.1 \\
$240^{\circ} \mathrm{C}$ & 3.51 & 9.85 & 5.21 & 20.8
\end{tabular}

for sample preservation and transportation. When the L-CDs were redispersed in water or ethanol, the emission peaks of samples prepared at higher temperatures moved very little, while the peaks of low-temperature samples exhibited a redshift of $\sim 25 \mathrm{~nm}$ (Figure S16). This might be a result of the difference in media polarity.

Fluorescence is well-known to be often quenched when aqueous dispersions with L-CDs are deposited on glass, silicon or plastic substrates (such as PET). L-CDs prepared by many teams could not emit light upon drying ${ }^{11,46,47}$. This shortcoming, explained by aggregation, significantly limits the wide applications of such materials. So far, only a few groups reported that their L-CDs were luminescent upon drying ${ }^{16,30,48}$. Based on previous research, one can conclude that luminescent L-CDs are very sensitive to their environment. When a material is dried, its close environment is destroyed, resulting in fluorescence quenching. This speculation is also supported by the above described shift of emission peak position in different liquid media, intensity changes in liquids with different $\mathrm{pH}$ and ionic strengths. Hence, to preserve the luminescence abilities of L-CDs in solid state, we prepared their composites with several polymers (such as PVP, PAA and PAM). The polymers acted as a matrix and kept the state of L-CDs similar to that in liquid media. Thus far, there have been several attempts to prepare polymer/L-CDs composites, however, the materials were reported to emit only the light characteristic of L-CDs themselves ${ }^{9,44,49,50}$. In contrast, the composites prepared in this study emitted different colors, even when the same L-CDs were used. In addition, samples prepared at different temperatures also exhibited different colors when incorporated into

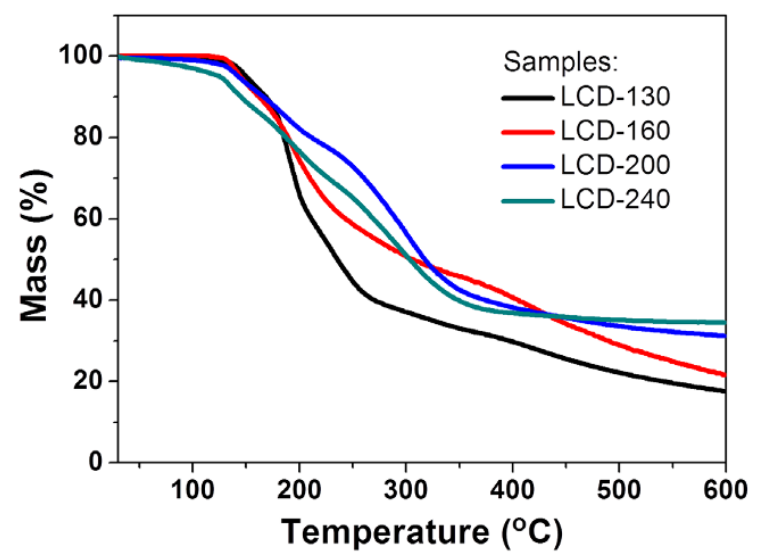

Figure $5 \mid$ TG curves of carbon dot solids fabricated at 130, 160, 200 and $240^{\circ} \mathrm{C}$. The higher the reaction temperature was, the larger residual mass was observed due to further carbonization. 


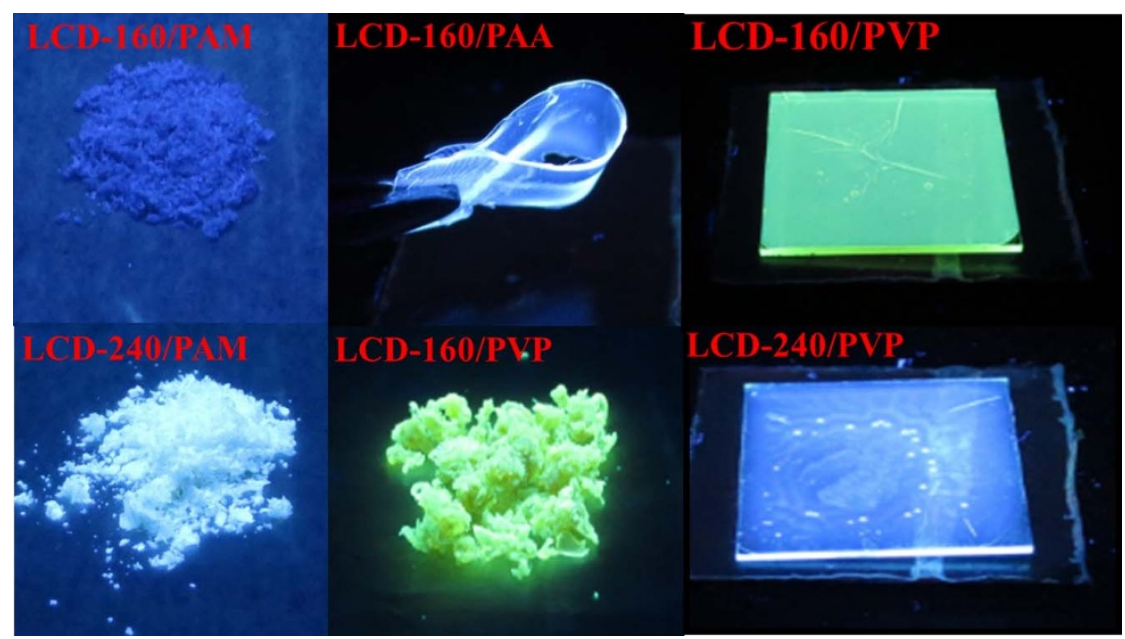

Figure 6 | Multicolor composites based on carbon dots with polymers: L-CDs/PAM blue and white phosphors, L-CDs/PAA and L-CDs/PVP composites, L-CDs/PVP composite films using L-CDs prepared at 160 and $240^{\circ} \mathrm{C}$.

the same polymer matrices. As shown in Figure 6, PAM incorporated with LCD-160 and LCD-240 displayed different colors, blue and white in a UV lamp box, respectively. PVP incorporated with the same L-CDs samples also exhibited different colors, green and blue. To our knowledge, this is the first report on L-CDs/polymer composites demonstrating several different colors. In addition, L-CDs with PVP could be spin-coated as thin films on glass, PET and other substrates. Flexible fluorescent films of L-CDs composites with PAA were also prepared. As the above described solid composites can be easily cut, polished or mold-formed as different shapes, they may be attractive materials for various potential applications, including LED, optical sensing, biomedical imaging, and even UV/laser protection for optical devices ${ }^{44,50}$. Note that all the composites tested here remained luminescent after at least as long as six months.

Ion sensing. Fluorescent carbon dot-based nanosensors have been undergoing rapid development lately. By detecting changes in fluorescence intensity caused by external physical or chemical stimuli, such sensors can detect metal ions, such as $\mathrm{Fe}^{3+}{ }^{9,10}$, $\mathrm{Hg}^{2+}{ }^{51-53}, \mathrm{Cu}^{2+}{ }^{22}$ and so on. Many efforts have been recently made to detect $\mathrm{Be}^{2+}$ ions by means of organic materials $\mathrm{s}^{54-56}$. In spite of good performance, the cost is still very high as such materials are prepared through complex synthetic routes and with quite low yields. Moreover, some of them are complex dyes causing potential environmental pollution. If the luminescence intensity of $\mathrm{L}-\mathrm{CD}$ s prepared in this study can be greatly affected by $\mathrm{Be}^{2+}$ ions in water, they might be a good candidate for the detection of $\mathrm{Be}^{2+}$ ions. Moreover, both the precursors and the prepared L-CDs are environmentally friendly and the preparation method is simple. That is why LCD-160 was tested for the detection of $\mathrm{Be}^{2+}$ ions. The detection capability was still good when $\mathrm{Be}^{2+}$ concentration was below $0.1 \mathrm{mM}$ (Figure 7a) and the calculated detection limit was $23.3 \mu \mathrm{M}$ (Figure $7 \mathrm{a}$ inset) according to the Stern-Volmer equation $^{53}$. Consequently, L-CDs can be considered as a candidate material for the detection of beryllium ions, especially if the detection limit is further improved.

Furthermore, we found that our L-CDs could be utilized as a water-sensor reagent for the detection of $\mathrm{Fe}^{3+}$ in water (Figure $7 \mathrm{~b}$ ). The fluorescence of the L-CDs could be quenched by $\mathrm{Fe}^{3+}$ ions due to the special coordination interaction between $\mathrm{Fe}^{3+}$ and the L-CDs. Fluorescence quenching may contribute to nonradiative electrontransfer that involves partial transfer for an electron in the excited state to the $\mathrm{d}$ orbital of $\mathrm{Fe}^{3+9}$. In addition, we also investigated the fluorescence quenching effect of other metal ions $\left(\mathrm{K}^{+}, \mathrm{Zn}^{2+}, \mathrm{Al}^{3+}\right.$, $\mathrm{Mn}^{2+}, \mathrm{Mg}^{2+}, \mathrm{Cu}^{2+}, \mathrm{Na}^{+}$and $\mathrm{Ca}^{2+}$ ) on L-CDs. Different metal ions with a concentration of $10^{-3} \mathrm{M}$ were added into a L-CDs dispersion $(10 \mathrm{~mL})$. Different quenching degrees were observed. Though $\mathrm{Cu}^{2+}$ also displayed a relatively strong quenching effect, the most promising results were demonstrated by $\mathrm{Be}^{2+}$ and $\mathrm{Fe}^{3+}$ ions (Figure 7c).

\section{Discussion (Conclusion)}

In conclusion, luminescent carbon dots with different surface states were prepared via a facile and high-output hydrothermal method. By controlling reaction temperature, materials with different surface density of amino-groups, and both excitation-dependent and independent luminescence, could be prepared. The latter excitation dependence or independence was found to depend on the surface states related to the presence (or absence) of surface groups such as carboxyl, hydroxyl and others: upon their complete passivation with surface amino-groups, the prepared materials demonstrated excitation-independent luminescence. The achieved quantum yield of luminescence was as high as $44.7 \%$. The prepared carbon dots were used to prepare composites with various polymers, giving products with different emission light, blue, green or white, depending on polymers and carbon dots used. Such composites are potentially attractive for LEDs, bioimaging and UV protection for optical devices. The carbon dots with excitation-independent luminescence could also be utilized as a label for the detection of toxic $\mathrm{Be}^{2+}$ in water with a detection limit as low as $23.3 \mu \mathrm{M}$.

\section{Methods}

Preparation of L-CDs. Carbon dots were prepared as follows: certain amounts (see details in Table S17) of citric acid and urea were dissolved in distilled water (25 mL). The mixture was transferred to a Teflon autoclave and heated at 130, 160, 200 and $240^{\circ} \mathrm{C}$ for $6 \mathrm{~h}$, the samples being further denoted as LCD-130, LCD-160, LCD-200 and LCD-240, respectively. After that, the reactor was cooled to room temperature naturally. The obtained samples were centrifuged at $10,000 \mathrm{rpm}$ for $10 \mathrm{~min}$ to remove larger particles. Then, the transparent blue (or brown) dispersion was dialyzed against pure water through a dialysis membrane $(\mathrm{Da}=1000)$ for $24 \mathrm{~h}$ to remove nonreacted ions and small particles. Finally, the resultant dispersion was dried in vacuum at $70^{\circ} \mathrm{C}$ for $72 \mathrm{~h}$. The final products were polymer-like materials, and the highest production yield was $\sim 67 \%$. The detailed reaction conditions, molar ratio of precursors and corresponding quantum yields of the samples are listed in Table S18.

Preparation of Polymer/L-CDs composites. Polymers (such as polyvinyl pyrrolidone (PVP), polyacrylamide (PAM) and poly acrylic acid (PAA)) were dissolved in ethanol and $20 \mu \mathrm{L}$ of a chosen L-CDs dispersion was added into the polymer solution. The mixture was then dried in vacuum at $60^{\circ} \mathrm{C}$ for $24 \mathrm{~h}$. Either powders or films were obtained depending on the nature of polymers used.

Material Characterization. X-ray diffraction (XRD) patterns were recorded on a multipurpose XRD system D8 Advance from Bruker with a $\mathrm{Cu} \mathrm{K} \alpha$ radiation. Raman-shift spectra were measured on a JY HR800 tool with laser excitation of 

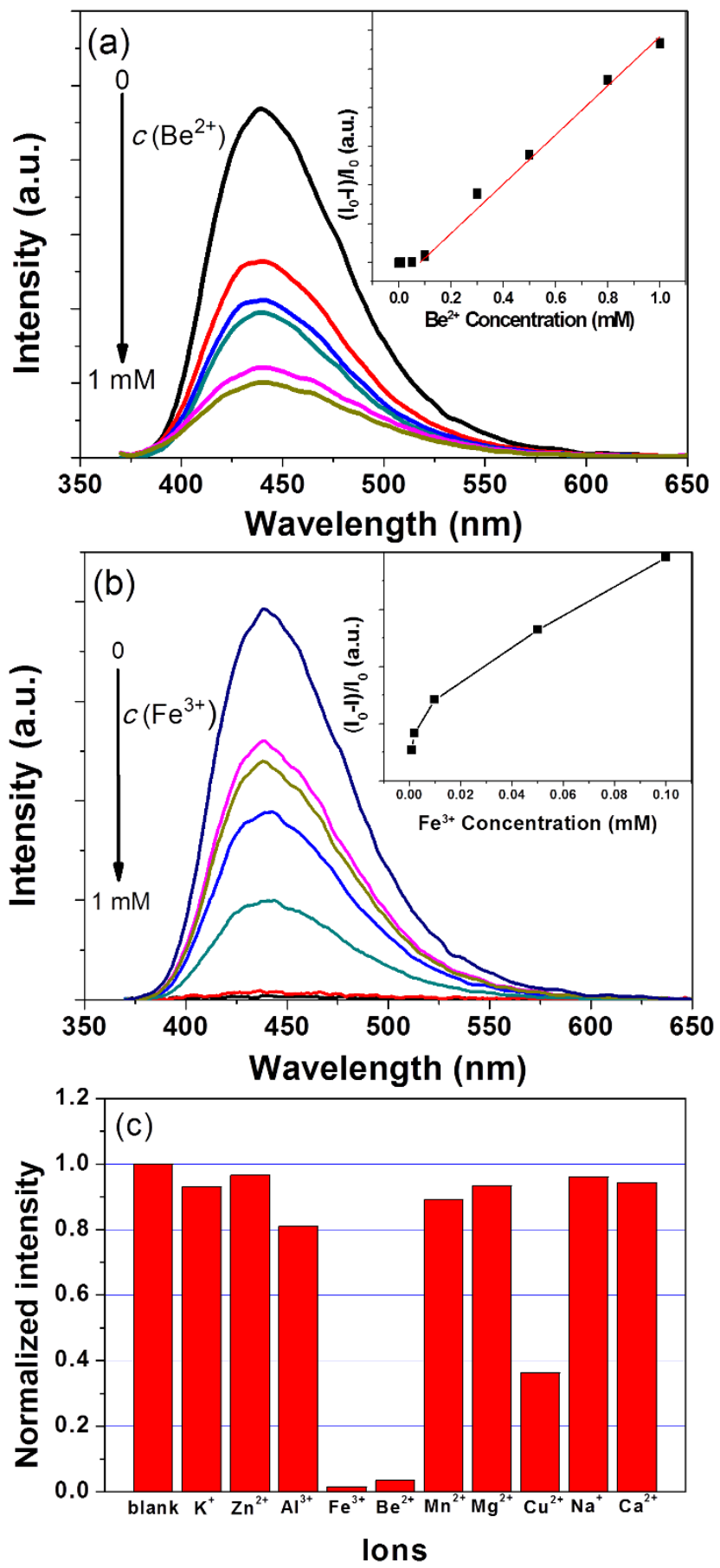

Figure $7 \mid$ (a) Typical PL quenching of LCD-160 in the presence of $\mathrm{Be}^{2+}$ ions; (b) Typical PL quenching of LCD-160 in the presence of $\mathrm{Fe}^{3+}$ ions; both the insets are concentration-dependent fluorescence response.

(c) Performance of sensors based on prepared carbon dots: comparison of fluorescence intensities in the absence and presence of different metal ions.

$488 \mathrm{~nm}$. UV-Vis absorption spectra were obtained using a Shimadzu $2660 \mathrm{UV}-\mathrm{V}$ is spectrophotometer, and PL spectra were measured with a Varian Cary Eclipse instrument. High-resolution transmission electron microscopy (HRTEM) images were taken on a FEI Tecnai F30 S-TWIN. Fluorescence lifetimes were measured using a Horiba FM-4P time-corrected single photon counting system. FTIR spectra were recorded with a Tensor-27 spectrometer. X-ray photoelectron spectroscopy (XPS) analysis was carried out on an ARL-9800 instrument with a monochromatic X-ray source $\mathrm{Al} \mathrm{K} \alpha$ excitation (1486.6 eV). Binding energy calibration was based on the $\mathrm{C} 1 \mathrm{~s}$ peak at $284.6 \mathrm{eV}$. TG curves were recorded with a Pyris-1-TGA system from PerkinElmer. Sample images were taken by a digital camera under illumination from a UV lamp.
Quantum yield measurements. Following a typical procedure from the literature, quinine sulfate (in $0.1 \mathrm{M} \mathrm{H}_{2} \mathrm{SO}_{4}$ as a solvent, with the quantum yield of 54\%) was chosen as a reference. The samples' QY was determined by means of the slope method, comparing the integrated PL intensity and the absorbance values of the samples with that of the reference. Then the QY was calculated according to the equation: $\varphi_{X}=\varphi_{r e}\left(K_{X} / K_{r e}\right)\left(\eta_{X} / \eta_{r e}\right)^{2}$ Where $\varphi$ is the QY, $K$ is the slope determined from the curves and $\eta$ is the refractive index. The subscripts "re" and " $\mathrm{x}$ " refer to the reference and the samples, respectively. Because of low material concentration in the samples, $\eta_{\mathrm{x}} / \eta_{\mathrm{st}}$ was considered as equal to 1 .

1. Yu, W. et al. Experimental Determination of the Extinction Coefficient of CdTe, CdSe, and CdS Nanocrystals. Chem. Mater. 15, 2584-2860 (2003).

2. Peng, Z. et al. Formation of High-Quality CdTe, CdSe, and CdS Nanocrystals Using CdO as Precursor. J. Am. Chem. Soc. 123, 183-184 (2000).

3. Li, H. et al. Carbon nanodots: synthesis, properties and applications. J. Mater. Chem. 22, 24230-24253 (2012).

4. Wang, F. et al. Highly Luminescent Organosilane-Functionalized Carbon Dots. Adv. Funct. Mater. 21,1027-1031 (2011).

5. Li, H. et al. Water-Soluble Fluorescent Carbon Quantum Dots and Photocatalyst Design. Angew. Chem. Int. Ed. 49, 4430-4434 (2010).

6. Wang, F. et al. White light-emitting devices based on carbon dots' electroluminescence. Chem. Commun. 47, 3502-3504 (2011).

7. Wang, F. et al. Synthesis of direct white-light emitting carbogenic quantum dots. Chem. Commun. 46, 3309-3311 (2010).

8. Chandra, S. et al. Luminescent S-doped carbon dots: an emergent architecture for multimodal applications. J. Mater. Chem. B 1, 2375-2382 (2013).

9. Zhu, S. et al. Highly Photoluminescent Carbon Dots for Multicolor Patterning, Sensors, and Bioimaging. Angew. Chem. Int. Ed. 52, 3953-3957 (2013)

10. Qu, S. et al. Ratiometric fluorescent nanosensor based on water soluble carbon nanodots with multiple sensing capacities. Nanoscale 5, 5514-5518 (2013).

11. Kong, B. et al. Carbon Dot-Based Inorganic-Organic Nanosystem for Two Photon Imaging and Biosensing of $\mathrm{pH}$ Variation in Living Cells and Tissues. $A d v$. Mater. 24, 5844-5848 (2012).

12. Yang, Z. et al. Intrinsically fluorescent carbon dots with tunable emission derived from hydrothermal treatment of glucose in the presence of monopotassium phosphate. Chem. Commun. 47, 11615-11617 (2011).

13. Zhou, J. et al. Electronic structure and luminescence center of blue luminescent carbon nanocrystals. Chem. Phys. Lett. 474, 320-324 (2009).

14. Bourlinos, A. et al. Surface Functionalized Carbogenic Quantum Dots. Small 4, 455-458 (2008).

15. Zhou, J. et al. An Electrochemical Avenue to Blue Luminescent Nanocrystals from Multiwalled Carbon Nanotubes (MWCNTs). J. Am. Chem.Soc. 129, 744-745 (2007).

16. Sun, Y. et al. Quantum-Sized Carbon Dots for Bright and Colorful Photoluminescence. J. Am. Chem. Soc. 128, 7756-7757 (2006).

17. Anilkumar, P. et al. Toward quantitatively fluorescent carbon-based "quantum" dots. Nanoscale 3, 2023-2027 (2011).

18. Wang, X. et al. Photoinduced electron transfers with carbon dots. Chem. Commun. 25, 3774-3776 (2009).

19. Dong, Y. et al. Polyamine-functionalized carbon quantum dots for chemical sensing. Carbon 50, 2810-2815 (2012).

20. Qu, S. et al. A Biocompatible Fluorescent Ink Based on Water-Soluble Luminescent Carbon Nanodots. Angew. Chem. Int. Ed. 51, 12215-12218 (2012).

21. Wu, Z. et al. One-pot hydrothermal synthesis of highly luminescent nitrogendoped amphoteric carbon dots for bioimaging from Bombyx mori silk - natural proteins. J. Mater. Chem. B 1, 2868-2873 (2013).

22. Liu, S. et al. Hydrothermal Treatment of Grass: A Low-Cost, Green Route to Nitrogen-Doped, Carbon-Rich, Photoluminescent Polymer Nanodots as an Effective Fluorescent Sensing Platform for Label-Free Detection of Cu(II) Ions. Adv. Mater. 24, 2037-2041 (2012).

23. Zhai, X. et al. Highly luminescent carbon nanodots by microwave-assisted pyrolysis. Chem. Commun. 48, 7955-7957 (2012).

24. Yang, Y. et al. One-step synthesis of amino-functionalized fluorescent carbon nanoparticles by hydrothermal carbonization of chitosan. Chem. Commun. 48, 380-382 (2012).

25. Pan, D. et al. Observation of $\mathrm{pH}-$, solvent-, spin-, and excitation-dependent blue photoluminescence from carbon nanoparticles. Chem. Commun. 46, 3681-3683 (2010).

26. Hu, S. et al. One-step synthesis of fluorescent carbon nanoparticles by laser irradiation. J. Mater. Chem. 19, 484-488 (2009).

27. Sun, Y. et al. Doped Carbon Nanoparticles as a New Platform for Highly Photoluminescent Dots. J. Phys. Chem. C 112, 18295-18298 (2008).

28. Bao, L. et al. Electrochemical Tuning of Luminescent Carbon Nanodots: From Preparation to Luminescence Mechanism. Adv. Mater. 23, 5801-5806 (2011).

29. Shang, J. et al. The Origin of Fluorescence from Graphene Oxide. Sci. Rep. 2, 792 (2012).

30. Yu, P. et al. Temperature-Dependent Fluorescence in Carbon Dots. J. Phys. Chem. C 116, 25552-25557 (2012).

31. Zhu, S. et al. Surface Chemistry Routes to Modulate the Photoluminescence of Graphene Quantum Dots: From Fluorescence Mechanism to Up-Conversion Bioimaging Applications. Adv. Funct. Mater. 22, 4732-4740 (2012). 
32. Peng, H. et al. Simple Aqueous Solution Route to Luminescent Carbogenic Dots from Carbohydrates. Chem. Mater. 21, 5563-5565 (2009).

33. Iijima, S. Helical microtubules of graphitic carbon. Nature 354, 56-58 (1991).

34. Pan, D. et al. Hydrothermal Route for Cutting Graphene Sheets into BlueLuminescent Graphene Quantum Dots. Adv. Mater. 22, 734-738 (2010).

35. Zheng, H. et al. Enhancing the luminescence of carbon dots with a reduction pathway. Chem. Commun. 47, 10650-10652 (2011).

36. Zeng, H. et al. Blue Luminescence of $\mathrm{ZnO}$ Nanoparticles Based on NonEquilibrium Processes: Defect Origins and Emission Controls. Adv. Funct. Mater. 20, 561-572 (2010).

37. Mao, X. J. et al. Study on the fluorescence characteristics of carbon dots. Spectrochim. Acta A 75, 553-557 (2010).

38. Sun, W. et al. Study on fluorescence properties of carbogenic nanoparticles and their application for the determination of ferrous succinate. J. Lumin. 130, 1463-1469 (2010).

39. Gao, M. et al. A surfactant-assisted redox hydrothermal route to prepare highly photoluminescent carbon quantum dots with aggregation-induced emission enhancement properties. Chem. Commun. 49, 8015-8017 (2013).

40. Zhao, Q. et al. Facile preparation of low cytotoxicity fluorescent carbon nanocrystals by electrooxidation of graphite. Chem. Commun. 41, 5116-5118 (2008).

41. Fang, Y. et al. Easy Synthesis and Imaging Applications of Cross-Linked Green Fluorescent Hollow Carbon Nanoparticles. ACS Nano 6, 400-409 (2011).

42. Tang, L. et al. Deep Ultraviolet Photoluminescence of Water-Soluble SelfPassivated Graphene Quantum Dots. ACS Nano 6, 5102-5110 (2012).

43. Zhang, R. et al. Preparation of high-quality biocompatible carbon dots by extraction, with new thoughts on the luminescence mechanisms. Nanotechnology 24, 225601 (2013).

44. Krysmann, M. J. et al. Formation mechanism of carbogenic nanoparticles with dual Photoluminescence emission. J. Am. Chem.Soc. 134, 747-750 (2012).

45. Zhang, P. et al. A facile and versatile approach to biocompatible "fluorescent polymers" from polymerizable carbon nanodots. Chem. Commun. 48, 10431-10433 (2012)

46. Qiao, Z. et al. Commercially activated carbon as the source for producing multicolor photoluminescent carbon dots by chemical oxidation. Chem. Commun. 46, 8812-8814 (2010).

47. Wang, J. et al. Amphiphilic Egg-Derived Carbon Dots: Rapid Plasma Fabrication, Pyrolysis Process, and Multicolor Printing Patterns. Angew. Chem. Int. Ed. 51, 9297-9301 (2012).

48. Wang, F. et al. One-Step Synthesis of Highly Luminescent Carbon Dots in Noncoordinating Solvents. Chem. Mater. 22, 4528-4530 (2010).

49. Deng, Y. et al. Long lifetime pure organic phosphorescence based on water soluble carbon dots. Chem. Commun. 49, 5751-5753 (2013).

50. Xie, Z. et al. Organic-Inorganic Hybrid Functional Carbon Dot Gel Glasses. Adv. Mater. 24, 1716-1721 (2012)
51. Zhou, L. et al. Carbon nanodots as fluorescence probes for rapid, sensitive, and label-free detection of $\mathrm{Hg}^{2+}$ and biothiols in complex matrices Chem. Commun. 48, 1147-1149 (2012).

52. Wu, D. et al. Preparation of photoluminescent carbon nanodots by traditional Chinese medicine and application as a probe for $\mathrm{Hg}^{2+}$. Anal. Methods. $\mathbf{5}$, 3023-3027 (2013).

53. Lu, W. et al. Economical, Green Synthesis of Fluorescent Carbon Nanoparticles and Their Use as Probes for Sensitive and Selective Detection of Mercury(II) Ions. Anal. Chem. 84, 5351-5357 (2012).

54. Peng, R. et al. Highly sensitive and selective detection of beryllium ions using a microcantilever modified with benzo-9-crown-3 doped hydrogel. Analyst 137, 1220-1224 (2012).

55. Keizer,T. et al. Designer Ligands for Beryllium. J. Am. Chem. Soc. 126, 9484-9485 (2004).

56. Hosseini, M. et al. Fluorescence "Turn-On" chemosensor for the selective detection of beryllium Spectrochim. Acta A. 83, 161-164 (2011).

\section{Acknowledgments}

This work was financially supported by the National Basic Research Program of Chin (2014CB931700), NSFC (61222403, 11274173), the Doctoral Program Foundation of China (20123218110030), and the Opened Fund of the State Key Laboratory on Integrated Optoelectronics (IOSKL2012KF06).

\section{Author contributions}

X.M.L. and Y.L.L. conceived the experiements; S.L.Z. performed the calculations; X.M.L., S.A.K. and H.B.Z. prepared the manuscript. All authors reviewed the manuscript.

\section{Additional information}

Supplementary information accompanies this paper at http://www.nature.com/ scientificreports

Competing financial interests: The authors declare no competing financial interests.

How to cite this article: Li, X.M., Zhang, S.L., Kulinich, S.A., Liu, Y.L. \& Zeng, H.B. Engineering surface states of carbon dots to achieve controllable luminescence for solid-luminescent composites and sensitive $\mathrm{Be}^{2+}$ detection. Sci. Rep. 4, 4976; DOI:10.1038/ srep04976 (2014).

(c) (1) This work is licensed under a Creative Commons Attribution-NonCommercialBY SA ShareAlike 3.0 Unported License. The images in this article are included in the article's Creative Commons license, unless indicated otherwise in the image credit; if the image is not included under the Creative Commons license, users will need to obtain permission from the license holder in order to reproduce the image. To view a copy of this license, visit http://creativecommons.org/licenses/by-nc-sa/3.0/ 